\title{
EXPLOITING TRANSMISSION SPATIAL DIVERSITY IN FREQUENCY SELECTIVE SYSTEMS WITH FEEDBACK CHANNEL
}

\author{
Antonio Pascual Iserte, Ana I. Pérez-Neira, Miguel A. Lagunas Hernández \\ Department of Signal Theory and Communications Telecommunications Technological Center of Catalonia \\ Polytechnic University of Catalonia (UPC) CTTC - Edifici NEXUS I \\ C/ Jordi Girona 1-3 (Campus Nord UPC - mòdul D5), 08034 Barcelona (SPAIN) \\ e-mail:\{tonip,anuska\}@gps.tsc.upc.es, m.a.lagunas@cttc.es
}

\begin{abstract}
In this paper we address the design of a multiple transmit antenna system in which the Channel State Information (CSI) at the transmitter is not perfect. Two different approaches are analyzed: one based on the Minimization of the Mean Square Error (MMSE) and the other based on the application of the Maximum Likelihood Sequence Estimation (MLSE). In both cases a Bayesian criterion is used in order to take into account the error between the CSI and the real channel. Finally, some simulation results and conclusions are provided, showing which is the gain of these approaches when the error between the CSI and the real channel is either Gaussian or uniform, where this last case corresponds to a quantization of the channel time response in order to transmit the CSI through a feedback channel from the receiver to the transmitter.
\end{abstract}

\section{INTRODUCTION}

Spatial diversity is an efficient method so as to combat the impairments present in the wireless channel. In cellular communications or Wireless LAN's the receive antenna diversity is not attractive for the downlink channel because the mobile station should be equipped with multiple receive antennas. For this reason, the use of transmit antenna diversity for the downlink is more desirable.

Existing transmission schemes for exploiting the potential offered by transmit antenna arrays are generally concerned with increasing the diversity order. There are several examples of such techniques, such as the delay diversity strategy, a special case of a more general solution presented in [1]. Other possible approaches that increase the diversity order consist in the application of spacetime coding, technique presented in works such as [2] and [3].

Space-time codes do not exploit channel knowledge at the transmitter. Information about the channel, if available, should be used to improve the performance by means of optimal terminal filtering. It can be shown that under a zero forcing criterion, the maximization of the Signal to Noise Ratio (SNR) results in a decoupled or spatially scalable solution where each transmit branch can be designed independently. Fig. 1 shows a generalized architecture that allows a normalization of the filters dynamic, while the beamforming weights $\left\{w_{q}\right\}_{q=1}^{Q}$ are in charge of adjusting the transmit power. Modulation can be seen as a quantization process and its effects can be studied as quantization noise. This quantization at the output of each filter avoids instabilities, and so, IIR designs could also be used. By departing from this architecture,

This work was partially supported by the the Spanish Government (CICYT) TIC2001-2356-C02-01, FIT-070000-2000-649 (MEDEA+ UniLAN); and the Catalan Government 2001Fl 00714, 2001SGR 00268.

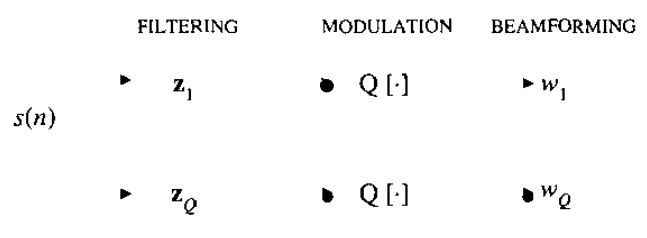

Fig. 1. Transmission diversity architecture.

a clear trade-off between optimality and crucial aspects like robustness, practicality, soft-degradation of the QoS, reliability, etc. can be easily taken into account. Concerning practical considerations, partial and quantized Channel State Information (CSI) at the transmitter can be introduced in a natural way by adapting, for instance, each complex weight $w_{q}$ to the strongest channel path at each branch or, if a controlled unitary dynamic is desired, just by compensating the phase of the strongest path. To sum up, robustness [4] implies insensitivity to deviations from the theoretical assumptions, being the imperfect CSI one of the possible sources of deviation.

So far we have commented on transmitter design based either on coding or linear processing depending on the channel knowledge, and how imperfections or bad knowledge can be taken into account by resorting to robust architectures. Another alternative existing in the literature for incorporating bad channel knowledge in transmit space-time processing is by means of a Bayesian point of view, that is, modeling the side channel information using a purely statistical approach. Previous and related work includes the performance analysis of [5] for flat fading channels or the design proposed in [6] for OFDM systems. In [7] the benefits of transmit beamforming and orthogonal space-time block coding for flat fading channels are combined. [8] considers the error in the CSI from a MAXMIN point of view different from the Bayesian one. In this paper we analyze the general case of a frequency selective channel and propose two space-time processing solutions that follow the Bayesian approach in order to incorporate the error in the CSI. Simulations compare the proposed techniques with spacetime processing designs that assume perfect CSI and schemes that do not need CSI such as delay diversity. Although the Bayesian approach will either result in spatially non-scalable solutions, or non-robust when the statistical assumptions are not true, it serves as a useful benchmark to analyze and compare the commented robust architectures. 


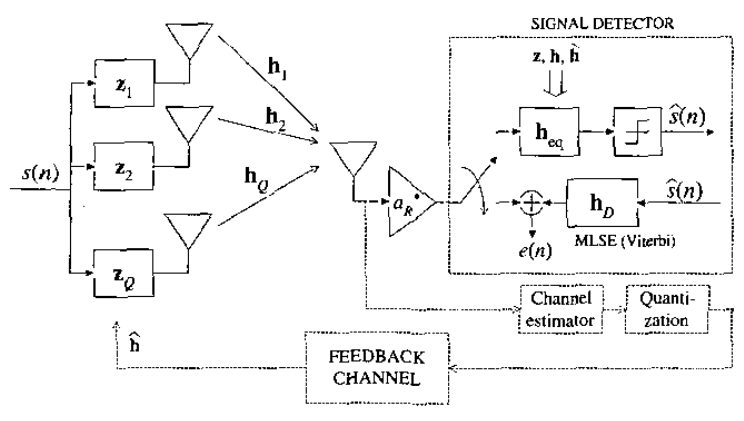

Fig. 2. General scheme for transmit diversity with feedback channel and imperfect CSI at the transmitter.

\section{PRE-FILTERING WITH FEEDBACK CHANNEL}

In this section we focus our attention on the case of a real system in which a digital feedback channel is implemented from the receiver to the transmitter. The single-antenna receiver is responsible for estimating the channel, quantize this estimate, code it into a digital format and send it to the transmitter via the feedback channel. By means of this, the transmitter has an estimate of the channel, possibly imperfect CSI. In this work we exploit this CSI in order to design linear filters at the transmitter from a Bayesian point of view and without forcing the design to be spatially scalable. The general scheme is presented in Fig. 2. Our goal is to design the filters $\left\{\mathbf{z}_{q}\right\}_{q=1}^{Q}$ for the $Q$ transmit antennas taking into account the imperfections in the CSI at the transmitter.

\subsection{System and Signal Models}

Let us consider a frequency selective channel with $Q$ transmit antennas, where each of the channels in the Multi-Input-SingleOutput (MISO) link has $L$ taps. $\mathbf{h}_{q}=\left[h_{1}^{(q)} h_{2}^{(q)} \cdots h_{L}^{(q)}\right]^{T}$ represents the time impulse response for the $q$ th transmit antenna. It is possible to collect all these time impulse responses in a single vector $\mathbf{h}$ by means of this notation: $\mathbf{h}=\left[\mathbf{h}_{1}^{T} \mathbf{h}_{2}^{T} \cdots \mathbf{h}_{Q}^{T}\right]^{T}$, where the number of components of $\mathbf{h}$ is $K=Q L$. The channel is modeled as a complex random Gaussian vector, where its covariance matrix $\mathbf{R}_{h}$ collects the spatial correlation and the power delay profile of the channel. In case that there is a direct line of sight, then these random vector would have a certain mean $\mathbf{m}$ different from zero. Therefore, the Probability Density Function (PDF) of the channel follows the statistical law: $\mathbf{h} \sim G\left(\mathbf{m}, \mathbf{R}_{h}\right)$.

Our goal is to design the transmit filters while considering very simple detectors at the receiver. As it is seen in Fig. 2, two possible receivers are considered: a symbol-by-symbol detector and a Maximum Likelihood Sequence Detector (MLSE) based on the application of the Viterbi algorithm. Let $\mathbf{z}_{q}=\left[z_{1}^{(q)} z_{2}^{(q)} \cdots z_{M}^{(q)}\right]^{T}$ be the time $M$-taps impulse response of the $q$ th transmit filter. Once again, it is possible to represent in a single vector $\mathbf{z}$ all these filters: $\mathbf{z}=\left[\mathbf{z}_{1}^{T} \mathbf{z}_{2}^{T} \cdots \mathbf{z}_{Q}^{T}\right]^{T}$. In the design of the transmit filters, the following transmit power constraint must be fulfilled:

$$
\|\mathrm{z}\|^{2}=\mathrm{z}^{H} \mathrm{z}=P_{t}
$$

At the transmitter side, only a channel estimate $\widehat{\mathbf{h}}$ or partial CSI is available. $\epsilon$ represents the error between the channel estimate $\widehat{\mathbf{h}}$ and the real channel realization $\mathbf{h}: \widehat{\mathbf{h}}=\mathbf{h}+\epsilon$. In the considered system, this channel error is due to the own estimation process at the receiver and/or the quantization of the channel estimate so that it can be transmitted through the feedback channel from the receiver to the transmitter. In general, we model this error statistically by means of its PDF, which is assumed to be known: $f_{\epsilon}(\epsilon)$. In case that no quantization is carried out, then the statistics of the error would usually correspond to a Gaussian PDF, whereas in case that only quantization is considered, the PDF would be uniform. By making use of this notation, it is possible to formulate the PDF of $\widehat{\mathbf{h}}$ conditioned to the real channel realization $\mathbf{h}$ as follows: $f_{\widehat{\mathbf{h}} \mid \mathbf{h}}(\widehat{\mathbf{h}} \mid \mathbf{h})=f_{\epsilon}(\widehat{\mathbf{h}}-\mathbf{h})$.

\section{SYSTEM DESIGNS}

In this section we present the two considered design strategies. The first one corresponds to a symbol-by-symbol detector based on the Minimum Mean Square Error (MMSE) criterion, whereas the other one makes use of a MLSE detector by means of the application of the Viterbi algorithm.

\subsection{Symbol-by-Symbol Detector}

When applying a symbol-by-symbol detector at the receiver, an adequate design criterion is MMSE, as it takes into account the noise power and also the signal distortion or Inter Symbol Interference (ISI). In case that the CSI was perfect, the equivalent channel impulse response at the receiver would be almost equalized. As this is not the case in a real scenario, we add a filter $h_{e q}$ at the receiver responsible for equalizing the residual ISI. When designing this filter, the MMSE criterion is considered and it is assumed that the real channel impulse response $\mathbf{h}$ is known at the receiver, as it is also assumed in [7]

$\xi(\mathbf{h}, \widehat{\mathbf{h}})$ is the Mean Square Error (MSE) for a concrete channel $\mathbf{h}$ and for a concrete collection of filters $\mathbf{z}(\widehat{\mathbf{h}})$ and gain factor at the receiver $a_{R}(\widehat{\mathbf{h}})$ :

$$
\xi(\mathbf{h}, \widehat{\mathbf{h}})=\left\|a_{R}^{*}(\widehat{\mathbf{h}}) \mathbf{H z}(\widehat{\mathbf{h}})-\mathbf{1}\right\|^{2}+\left|a_{R}(\widehat{\mathbf{h}})\right|^{2} \sigma_{w}^{2}
$$

where it is assumed that the symbols $s(n)$ are normalized so that $E\left\{|s(n)|^{2}\right\}=1, \mathbf{H}=\left[\mathbf{H}_{1} \mathbf{H}_{2} \cdots \mathbf{H}_{Q}\right]$ is a matrix containing all the Toeplitz convolution matrices $\left\{\mathbf{H}_{q}\right\}_{q=1}^{Q}$ corresponding to the $Q$ channels $\left\{\mathbf{h}_{q}\right\}_{q=1}^{Q}, \mathbf{1}$ is an all-zeros vector except a $\mathbf{1}$ in a position representing the desired temporal response of the equalized channel $a_{R}^{*} \mathbf{H z}$, and $\sigma_{w}^{2}$ represents the power of the Additive White Gaussian Noise (AWGN) at the receiver. The convolution matrix $\mathbf{H}_{q}$ is defined as the $(M+L-1) \times M$ dimensional Toeplitz matrix, where the first row is an all-zeros vector except the first element which is equal to $h_{1}^{(q)}$, and the first column is an all-zeros vector except the first $L$ elements which are equal to the vector $\mathbf{h}_{q}$.

Our goal is to design the filters so as to minimize the MSE averaged over the real channel statistics and the error statistics. This can be expressed as follows:

$$
\begin{aligned}
\xi & =\int f_{\widehat{\mathbf{h}}}(\widehat{\mathbf{h}}) \xi(\widehat{\mathbf{h}}) d \widehat{\mathbf{h}} \\
\xi(\widehat{\mathbf{h}}) & =E_{\mathbf{h} \mid \widehat{\mathbf{h}}}\{\xi(\mathbf{h}, \widehat{\mathbf{h}}) \mid \widehat{\mathbf{h}}\}=\int \xi(\mathbf{h}, \widehat{\mathbf{h}}) f_{\mathbf{h} \mid \widehat{\mathbf{h}}}(\mathbf{h} \mid \widehat{\mathbf{h}}) d \mathbf{h}
\end{aligned}
$$


As the filters $\mathbf{z}$ and gain factor $a_{R}$ depend only on the channel estimate $\widehat{\mathbf{h}}$, the minimization of $\xi$ is equivalent to the minimization of $\xi(\widehat{\mathbf{h}})$ subject to the transmit power constraint (1). The optimum solution corresponds to the following equalities:

$$
\begin{aligned}
\mathbf{z}(\widehat{\mathbf{h}}) & =\alpha\left(\mathbf{X}+\frac{\sigma_{w}^{2}}{P_{t}} \mathbf{I}\right)^{-1} \mathbf{M}^{H} \mathbf{1} \\
a_{R}(\widehat{\mathbf{h}}) & =\frac{\mathbf{1}^{H} \mathbf{M} \mathbf{z}}{\mathbf{z}^{H} \mathbf{X z}+\sigma_{w}^{2}}
\end{aligned}
$$

where $\alpha$ is a constant such that the transmit power constraint (1) is fulfilled, $\mathbf{X}(\widehat{\mathbf{h}})=E_{\mathbf{h} \mid \hat{\mathbf{h}}}\left\{\mathbf{H}^{H} \mathbf{H} \mid \widehat{\mathbf{h}}\right\}$ and $\mathbf{M}(\widehat{\mathbf{h}})=E_{\mathbf{h} \mid \hat{\mathbf{h}}}\{\mathbf{H} \mid \widehat{\mathbf{h}}\}$. In general it is difficult to obtain closed expressions of $\mathbf{X}$ and $\mathbf{M}$. In the Appendix we show how to obtain a closed expression for the case in which the error $\epsilon$ is assumed to be Gaussian.

\subsection{MLSE Detector}

It is also possible to use other kind of detectors with a higher computational load but with a better performance, such as the MLSE based on the Viterbi algorithm (see Fig. 2). This detector is the optimum one in case that the channel is known with no error at the receiver, which corresponds with our assumptions and as presented in [7]. The performance of this detector is directly related to the SNR, which is defined as follows:

$$
\operatorname{SNR}(\mathbf{h}, \widehat{\mathbf{h}})=\frac{1}{\sigma_{w}^{2}} \mathbf{z}^{H}(\widehat{\mathbf{h}}) \mathbf{H}^{H} \mathbf{H z}(\widehat{\mathbf{h}})
$$

where it is assumed that $E\left\{|s(n)|^{2}\right\}=1$ and $\mathbf{z}$ and $\mathbf{H}$ are defined as in the previous subsection. Our goal is to maximize the SNR averaged over the real channel statistics and the error statistics. This can be expressed as follows:

$$
\begin{aligned}
\operatorname{SNR} & =\int f_{\widehat{\mathbf{h}}}(\widehat{\mathbf{h}}) \operatorname{SNR}(\widehat{\mathbf{h}}) d \widehat{\mathbf{h}} \\
\operatorname{SNR}(\widehat{\mathbf{h}}) & =E_{\mathbf{h} \mid \widehat{\mathbf{h}}}\{\operatorname{SNR}(\mathbf{h}, \widehat{\mathbf{h}}) \mid \widehat{\mathbf{h}}\}=\int \operatorname{SNR}(\mathbf{h}, \widehat{\mathbf{h}}) f_{\mathbf{h} \mid \widehat{\mathbf{h}}}(\mathbf{h} \mid \widehat{\mathbf{h}}) d \mathbf{h}
\end{aligned}
$$

The maximization of the SNR is equivalent to the maximization of SNR $(\widehat{\mathbf{h}})$ subject to the power constraint (1). The solution to this optimization problem is found as an eigenvector problem:

$$
\begin{aligned}
\mathbf{z}(\widehat{\mathbf{h}}) & =\sqrt{P_{t}} \mathbf{u} \\
\lambda_{\max } \mathbf{u} & =\mathbf{X u}, \quad\|\mathbf{u}\|=1
\end{aligned}
$$

where the matrix $\mathbf{X}$ is defined as in the previous subsection: $\mathbf{X}(\widehat{\mathbf{h}})=$ $E_{\mathbf{h} \mid \hat{\mathbf{h}}}\left\{\mathbf{H}^{H} \mathbf{H} \mid \hat{\mathbf{h}}\right\}$. A closed expression of this matrix is presented in the Appendix when the error $\epsilon$ is assumed to be Gaussian.

It can be shown that this design criterion is equivalent to the minimization of the error power $P_{e}=E\left\{|e(n)|^{2}\right\}$. The equivalent time impulse response to be used when applying the Viterbi algorithm is: $\mathbf{h}_{D}=a_{R}^{*} \mathbf{H z}$. The gain factor $a_{R}$ is arbitrary and does not affect the performance of the system. Usually, $a_{R}$ is calculated so that the mean power at the input of the MLSE block is normalized to the unity. It must be said that in this section we assume that the Viterbi decoder admits any length of the equivalent response $\mathbf{h}_{D}$, and therefore, the complexity and computational load can be very high. Further work will analyze other kinds of detectors based on MLSE but with a lower computational complexity by means of a shortening of $\mathbf{h}_{D}$.

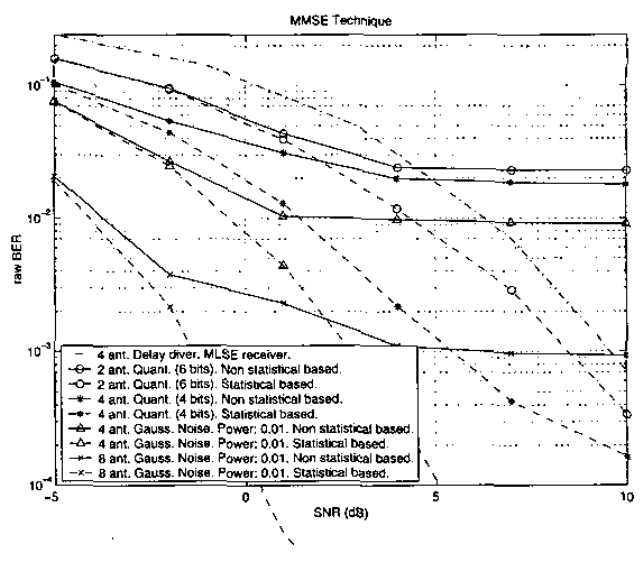

Fig. 3. Simulations results for the MMSE technique.

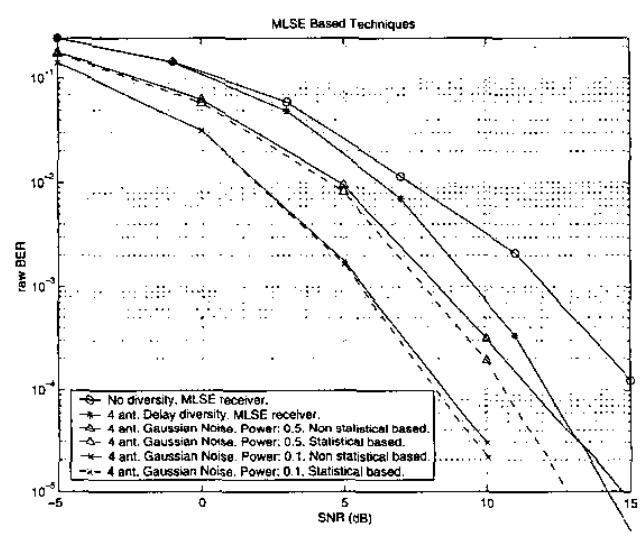

Fig. 4. Simulations results for the techniques based on a MLSE receiver.

\section{SIMULATION RESULTS AND CONCLUSIONS}

In this section we present some simulations that help to understand the benefits of using the designs presented in this work. We have simulated normalized channels $\left(E\left\{\left\|\mathbf{h}_{q}\right\|^{2}\right\}=1\right)$ with a delay spread of 3 symbol periods, an angular spread of $30^{\circ}$ and BPSK symbols. The length $L$ of the channel for the MMSE technique is 5 , whereas for the case of MLSE is 3 .

In Fig. 3 we present some results for the MMSE technique, in which the receiver is based on a symbol-by-symbol detector. The transmit filters $\mathbf{z}_{q}$ have 7 taps, whereas $\mathbf{h}_{e q}$ has 10 taps. In these simulations, we have always made use of the mathematical expressions presented in the Appendix, i. e., we have assumed that the error is Gaussian. Two different situations have been analyzed. The first one corresponds to an error which is actually Gaussian, whereas in the second case the error is due to the quantization of the channel impulse response, so, in this last case, the 
statistical model of the error does not correspond with the reality. We have simulated different powers of the Gaussian error and different number of bits to carry out the quantization (the number of bits in the figure represents the number of bits with which each tap of the channel is quantized). As it can be seen, the solution that does not take into account the error in the CSI and assumes that the channel estimate is perfect, is not able to decrease the BER although the SNR is increased, whereas in the case of the solution based on the Bayesian point of view (Eq. (4)), the BER decreases as the SNR increases. It can be also concluded that, although for the case of quantization the error model does not correspond with the real statistics, the Bayesian design is able to increase the performance in front of the non-statistical based solution, that is, the solution that assumes that the CSI is perfect.

In Fig. 4 the equivalent results for the MLSE technique are presented for 4 antennas and a Gaussian channel estimation error. The transmit filters have only 1 tap. We have also made comparisons between this solution, which needs CSI, and the delay diversity technique for 1 and 4 antennas, also detected by means of MLSE (Viterbi). Delay diversity is a linear precoding technique that does not need any CSI at the transmitter. As it can be seen, in this case the gains obtained by means of the Bayesian approach are less important than the ones obtained with the MMSE technique. The reason for it is that the Viterbi decoder is not sensitive to non-equalized channels and that the gains of mean SNR by means of the Bayesian approach are not extremely important and do not have a direct impact on the BER. It can be also concluded that, although the noise power in the CSI is very high and the quality of the channel estimate is very bad, the solution based on the MLSE technique permits increasing importantly the performance of the delay diversity scheme.

\section{APPENDIX}

In this Appendix we deduce the expressions corresponding to the matrices $\mathbf{M}(\widehat{\mathbf{h}})$ and $\mathbf{X}(\widehat{\mathbf{h}})$ when the error $\epsilon$ is assumed to be Gaussian with the following PDF: $\epsilon \sim G(0, \Sigma)$. Under this assumption it can be easily proved that $\hat{\mathbf{h}} \mid \mathbf{h} \sim G(\mathbf{h}, \mathbf{\Sigma})$ and $\mathbf{h} \mid \hat{\mathbf{h}} \sim$ $G(\mathbf{t}, \mathbf{C})$, where $\mathbf{t}$ and $\mathbf{C}$ are defined as follows:

$$
\begin{aligned}
\mathbf{C} & =\left(\mathbf{R}_{h}^{-1}+\mathbf{\Sigma}^{-1}\right)^{-1} \\
\mathbf{t} & =\mathbf{C}\left(\mathbf{R}_{h}^{-1} \mathbf{m}+\boldsymbol{\Sigma}^{-1} \widehat{\mathbf{h}}\right)
\end{aligned}
$$

Deduction of $M$ : let us write the vector $\mathbf{t}=E_{\mathbf{h} \mid \hat{\mathbf{h}}}\{\mathbf{h} \mid \widehat{\mathbf{h}}\}$ defined in (11) as follows: $\mathbf{t}=\left[\mathbf{t}_{1}^{T} \mathbf{t}_{2}^{T} \cdots \mathbf{t}_{Q}^{T}\right]^{T}$, where $\mathbf{t}_{q}=$ $\left[t_{1}^{(q)} t_{2}^{(q)} \cdots t_{L}^{(q)}\right]^{T}$ and $t_{l}^{(q)}=E_{\mathbf{h} \mid \hat{\mathbf{h}}}\left\{h_{l}^{(q)} \mid \widehat{\mathbf{h}}\right\}$. The matrix $\mathbf{M}(\widehat{\mathbf{h}})$ is defined as $\mathbf{M}=\left[\mathbf{M}_{1} \mathbf{M}_{2} \cdots \mathbf{M}_{Q}\right]$, where $\mathbf{M}_{q}=$ $E_{\mathbf{h} \mid \hat{\mathbf{h}}}\left\{\mathbf{H}_{q} \mid \widehat{\mathbf{h}}\right\}$ is the conditioned mean of the Toeplitz convolution matrix $\mathbf{H}_{q}$ associated to the channel corresponding to the $q$ th transmit antenna. The matrix $\mathrm{M}_{q}$ is the $(M+L-1) \times M$ Toeplitz convolution matrix, where the first row is an all-zeros vector except the first element which is equal to $t_{1}^{(q)}$, and the first column is an all-zeros vector except the first $L$ elements which are equal to the vector $\mathbf{t}_{q}$.

Deduction of $\mathrm{X}$ : let us write the matrix defined in (10) $\mathrm{C}=$ $E_{\mathbf{h} \mid \widehat{\mathbf{h}}}\left\{(\mathbf{h}-\mathbf{t})(\mathbf{h}-\mathbf{t})^{H} \mid \widehat{\mathbf{h}}\right\}$ as:

$$
\mathbf{C}=\left[\begin{array}{ccc}
\mathbf{C}^{(1)(1)} & \cdots & \mathbf{C}^{(1)(Q)} \\
\vdots & \ddots & \vdots \\
\mathbf{C}^{(Q)(1)} & \cdots & \mathbf{C}^{(Q)(Q)}
\end{array}\right]
$$

where $\mathbf{C}^{\left(q_{1}\right)\left(q_{2}\right)}=E_{\mathbf{h} \mid \hat{\mathbf{h}}}\left\{\left(\mathbf{h}_{q_{1}}-\mathbf{t}_{q_{1}}\right)\left(\mathbf{h}_{q_{2}}-\mathbf{t}_{q_{2}}\right)^{H} \mid \hat{\mathbf{h}}\right\}$. The matrix $\mathbf{X}(\widehat{\mathbf{h}})$ is defined as:

$$
\mathbf{X}=\left[\begin{array}{ccc}
\mathbf{R}^{(1)(1)} & \cdots & \mathbf{R}^{(1)(Q)} \\
\vdots & \ddots & \vdots \\
\mathbf{R}^{(Q)(1)} & \cdots & \mathbf{R}^{(Q)(Q)}
\end{array}\right]
$$

where $\mathbf{R}^{\left(q_{1}\right)\left(q_{2}\right)}=E_{\mathbf{h} \mid \widehat{\mathbf{h}}}\left\{\mathbf{H}_{q_{1}}^{H} \mathbf{H}_{q_{2}} \mid \widehat{\mathbf{h}}\right\}$ is a $M \times M$ matrix. Let $\left[\mathbf{R}^{\left(q_{1}\right)\left(q_{2}\right)}\right]_{m, n}$ represent the $(m, n)$ th component of $\mathbf{R}^{\left(q_{1}\right)\left(q_{2}\right)}$. It can be deduced that this component is calculated as follows:

$$
\begin{aligned}
& \sum_{i=1}^{L+m-n}\left[\mathbf{C}^{\left(q_{2}\right)\left(q_{1}\right)}\right]_{l, l+n-m}+t_{l+n-m}^{\left(q_{1}\right) *} t_{l}^{\left(q_{2}\right)}, m \leq n \\
& \sum_{l=1}^{L+n-m}\left[\mathbf{C}^{\left(q_{2}\right)\left(q_{1}\right)}\right]_{l+m-n, l}+t_{l}^{\left(q_{1}\right) *} t_{l+m-n}^{\left(q_{2}\right)}, \quad m>n
\end{aligned}
$$

By means of these equalities and relationships, the final expression of the matrix $\mathbf{X}(\widehat{\mathbf{h}})$ can be obtained.

\section{REFERENCES}

[1] G. W. Wornell and M. T. Trott, "Efficient Signal Processing Techniques for Exploiting Transmit Antenna Diversity on Fading Channels," IEEE Trans. on Signal Proc., vol. 45, no. 1, pp. 191-205, Jan. 1997.

[2] V. Tarokh, N. Seshadri, and A. R. Calderbank, "Space-Time Codes for High Data Rate Wireless Communication: Performance Criterion and Code Construction," IEEE Trans. on Inform. Theory, vol. 44, no. 2, pp. 744-765, March 1998.

[3] G. Ganesan and P. Stoica, "Space-Time Block Codes: a Maximum SNR Approach," IEEE Trans. on Inform. Theory, vol. 47, no. 2, pp. 1650-1656, May 2001.

[4] P-J. Huber, Robust Statistics, Wiley Series, 1981.

[5] Jinho Choi, "Performance Analysis for Transmit Antenna Diversity With/Without Channel Information," IEEE Trans. on Veh. Techn., vol. 51, no. 1, pp. 101-113, January 2002.

[6] F. Rey, M. Lamarca, and G. Vázquez, "A Joint TransmitterReceiver Design in MIMO Systems Robust to Channel Uncertainty for W-LAN Applications," in Proceedings IST Mobile Communic. Summit, Thessaloniki, June 2002.

[7] G. Jöngren, M. Skoglund, and B. Ottersten, "Combining Beamforming and Orthogonal Space-Time Block Coding," IEEE Trans. on Inform. Theory, vol. 48, no. 3, pp. 611-627, March 2002.

[8] D. P. Palomar, M. A. Lagunas Hernández, and John M. Cioff, "Optimum Linear Joint Transmit-Receive Processing for MIMO Channels with QoS Constraints," Submitted to IEEE Trans. on Signal Proc., 2002. 